\title{
Development of a risk scoring system for prognostication in HIV-related toxoplasma encephalitis
}

\author{
Yao Li ${ }^{1 \dagger}$, Yan-Ming Zeng ${ }^{1 \dagger}$, Min Liu', Yan-Qiu Lu', Xue-Yan Liu², Yu-Lin Zhang ${ }^{3}$, Zhong-Sheng Jiang ${ }^{4}$, \\ Tong-Tong Yang ${ }^{5}$, Yan Sun ${ }^{6}$, Ke Lan ${ }^{7}$ and Yao-Kai Chen ${ }^{1 *}$
}

\begin{abstract}
Background: This study aims to evaluate specific risk factors influencing prognosis of HIV-infected patients with toxoplasma encephalitis (TE) in order to develop a prognostic risk scoring system for them.

Methods: This is a six-center retrospective study of hospitalized HIV/TE patients. Data including six-week mortality after diagnosis, baseline characteristics, clinical features, laboratory tests and radiological characteristics of eligible patients were assimilated for risk model establishing.

Results: In this study, the six-week mortality among 94 retrospective cases was $11.7 \%$ (11/94). Seven specific risk factors, viz. time from symptom onset to presentation, fever, dizziness, CD4+ T-cell counts, memory deficits, patchy brain lesions, and disorders of consciousness were calculated to be statistically associated with mortality. A criterion value of ' 9 ' was selected as the optimal cut-off value of the established model. The AUC of the ROC curve of this scoring model was $0.976(p<0.001)$. The sensitivity and specificity of the risk scoring model was 100.0 and $86.9 \%$, respectively, which were 81.8 and $94.1 \%$ of this scoring model in the verification cohort, respectively.
\end{abstract}

Conclusions: The developed scoring system was established with simple risk factors, which also allows expeditious implementation of accurate prognostication, and appropriate therapeutic interventions in HIV-infected patients with TE.

Keywords: HIV, Toxoplasma encephalitis, Risk scoring system, Death, Retrospective study

\section{Background}

Toxoplasmosis is a zoonotic parasitic disease caused by Toxoplasma gondii infection [1]. In HIV-infected patients, seropositivity for Toxoplasma antibodies is as high as $10-40 \%$, and it is estimated that a third of those patients will eventually progress to toxoplasma encephalitis (TE) [2], particularly in those with CD4+ T-cell counts $<50$ cells $/ \mu \mathrm{L}$ [3]. Unfortunately, the poor prognosis of TE generally leads to death or disability in HIV-

\footnotetext{
* Correspondence: yaokaichen@hotmail.com

${ }^{\dagger}$ Yao Li and Yan-Ming Zeng contributed equally to this work.

${ }^{1}$ Division of Infectious Diseases, Chongqing Public Health Medical Center, 109 Baoyu Road, Shapingba, Chongqing, China

Full list of author information is available at the end of the article
}

infected patients. Even after anti-toxoplasma treatment, the mortality of HIV-associated TE during hospitalization is still as high as $29.9 \%$ [4]. Thus, early identification of high-risk cases at the diagnosis of TE is crucial for improving prognosis and reducing TEassociated mortality among HIV-infected patients.

Previous studies have reported on potential predictors of poor prognosis of central nervous system toxoplasmosis. For example, independent risk factors for death among HIV-infected patients with TE were acute kidney injury (AKI) and hyponatremia [5]. CD4+ T-cell counts and Glasgow Coma Scale (GCS) scores were independently associated with poor outcome (modified Rankin Scale $>2$ ) in HIV-infected patients with severe cerebral

(C) The Author(s). 2020 Open Access This article is licensed under a Creative Commons Attribution 4.0 International License, which permits use, sharing, adaptation, distribution and reproduction in any medium or format, as long as you give appropriate credit to the original author(s) and the source, provide a link to the Creative Commons licence, and indicate if changes were made. The images or other third party material in this article are included in the article's Creative Commons licence, unless indicated otherwise in a credit line to the material. If material is not included in the article's Creative Commons licence and your intended use is not permitted by statutory regulation or exceeds the permitted use, you will need to obtain permission directly from the copyright holder. To view a copy of this licence, visit http://creativecommons.org/licenses/by/4.0/ The Creative Commons Public Domain Dedication waiver (http://creativecommons.org/publicdomain/zero/1.0/) applies to the data made available in this article, unless otherwise stated in a credit line to the data. 
toxoplasmosis [6]. It is therefore apparent that there have been studies considering factors related to the risk of poor prognosis in TE, and that the reported factors are not specific, and are mainly restricted to specific laboratory tests. In addition, there is at present no risk stratification scoring model for TE among HIV-infected patients specifically designed to prognosticate outcomes. Therefore, the development of a user-friendly scoring model with clear outcome prognostication benefit, and a favorable predictive value, is urgently needed.

In this study, risk factors for death were analyzed using the data from 94 HIV-infected patients with TE. This retrospective cohort study aimed to determine the risk factors related to prognosis, and establish a simple scoring system based on baseline characteristics, clinical features, laboratory tests, and radiological characteristics, and subsequently to identify its effectiveness in a prospective validation cohort of HIV-infected patients with TE.

\section{Methods \\ Patients}

This was a six-center retrospective study involving hospitalized patients. One hundred fifty-six HIV-infected patients admitted to hospital from May 2013 to September 2019, and diagnosed with TE were eligible to participate in the present study. Six-week mortality after diagnosis was defined as the study outcome. Six-week is the time point at the end of anti-Toxoplasma therapy recommended by the National Institutes of Health (NIH) guidelines [7], and is a vital time point to assess the efficacy of the treatment, and adverse outcomes such as mortality [8]. Sixty-two patients were excluded from the retrospective cohort, due to the absence of outcome data, demographic characteristics, clinical features, laboratory test results, or radiological findings. Subsequently, the demographic and clinical data of 94 patients were used for the development of our scoring model. Forty-five patients who were admitted to hospital between October 2019 and March 2020 and with appropriate and available data were eligible to be included in our validation cohort.

\section{Standard protocol approvals, registrations, and patient consents}

The present study was approved by the institutional review board of Chongqing Public Health Medical Center (No. 2019-003-02-KY). The institutional review board waived the requirement for written informed consent, since the present study was retrospective and all patients' data were analyzed in anonymity.

The raw data may be requested from the first author and corresponding author, with administrative permissions of the review board of the Chongqing Public
Health Medical Center, first author and corresponding author.

\section{Data collection}

Six-week mortality after diagnosis, details of baseline characteristics, clinical features, laboratory tests, and radiological characteristics of 94 patients were collected, including: gender, age, ART initiation, time from symptom onset to presentation, headache, dizziness, fever, vomiting, memory deficits, cognitive impairment, disorders of consciousness, dysphasia, meningeal irritation signs, CD4+ T-cell counts, patchy CT/MRI lesions, and ring-enhancing $\mathrm{CT} / \mathrm{MRI}$ lesions.

\section{Statistical analysis}

All data analysis was executed using the Statistical Package for the Social Sciences (SPSS) Version 23.0 software (IBM-SPSS, Armonk, NY, USA) and MedCalc Version 18.9 (MedCalc Software Ltd., Ostend, Belgium). Univariate logistic regression analysis was performed to assess potential predictors of non-survival, and potential factors with $p$-values $<0.20$ were included in the multivariate stepwise forward logistic regression analysis for score assignment. A receiver operating characteristic (ROC) curve of this scoring model was then generated, and the area under the curve (AUC) of ROC curve was calculated to assess model accuracy. Also, a cut-off value with optimal sensitivity and specificity was identified in the validation cohort.

\section{Results}

\section{Patients}

The six-week mortality among 94 retrospective cases in the present study was $10.6 \%(10 / 94)$. As shown in Table 1, 38.3\% (36/94) of patients were aged $\geq 40$ years old, $74.5 \%$ (70/94) were male, around one thirds of the cohort had headaches (32/94), dizziness (31/ $94)$, or fever (32/94), and $25.5 \%(24 / 94)$ of cases had vomiting as a symptom at baseline. The study flow diagram is displayed in the supplementary information Figure 1.

\section{Univariate logistic regression analysis}

Sixteen variables, considered to be associated with nonsurvival in HIV-associated TE cases, were selected for univariate logistic regression analysis. The post-analysis results revealed that significant statistical differences existed among certain variables, viz. memory deficits, disorders of consciousness, dysphasia, and CD4+ T-cell counts between survivors and non-survivors $(p<0.05)$. Details were illustrated in Table 1. 
Table 1 Univariate logistical regression analysis

\begin{tabular}{|c|c|c|c|c|c|}
\hline Variable & Total & Death & Survival & $p$ & OR $(95 \% \mathrm{Cl})$ \\
\hline Age (years old): & 94 & & & 0.570 & $1.510(0.364,6.256)$ \\
\hline$<40$ & & $7 ;$ & $51 ;$ & & \\
\hline$\geq 40$ & & 3 & 33 & & \\
\hline Gender: & 94 & & & 0.259 & $3.393(0.407,28.297)$ \\
\hline Male; & & $9 ;$ & $61 ;$ & & \\
\hline Female & & 1 & 23 & & \\
\hline Fever: & 94 & & & 0.123 & $0.109(0.636,43.550)$ \\
\hline Yes; & & $1 ;$ & $31 ;$ & & \\
\hline No & & 9 & 53 & & \\
\hline Dizziness: & 94 & & & 0.136 & $0.500(0.604,41.392)$ \\
\hline Yes; & & $1 ;$ & 30 & & \\
\hline No & & 9 & 54 & & \\
\hline Headache: & 94 & & & 0.776 & $1.230(0.296,5.117)$ \\
\hline No; & & $3 ;$ & $29 ;$ & & \\
\hline Yes & & 7 & 55 & & \\
\hline Vomiting: & 94 & & & 0.732 & $1.286(0.305,5.426)$ \\
\hline No; & & $7 ;$ & $63 ;$ & & \\
\hline Yes & & 3 & 21 & & \\
\hline Memory deficits: & 94 & & & 0.030 & $4.500(1.157,17.500)$ \\
\hline No; & & $4 ;$ & $63 ;$ & & \\
\hline Yes & & 6 & 21 & & \\
\hline Cognitive impairment: & 94 & & & 0.133 & $3.950(0.657,23.749)$ \\
\hline No; & & $8 ;$ & $79 ;$ & & \\
\hline Yes & & 2 & 5 & & \\
\hline Disorders of consciousness: & 94 & & & 0.005 & $7.400(1.817,30.144)$ \\
\hline No; & & 5; & $74 ;$ & & \\
\hline Yes & & 5 & 10 & & \\
\hline Dysphasia: & 94 & & & 0.008 & $6.375(1.608,25.274)$ \\
\hline No; & & $4 ;$ & $68 ;$ & & \\
\hline Yes & & 6 & 16 & & \\
\hline Meningeal irritation signs: & 94 & & & 0.053 & $6.750(0.979,46.551)$ \\
\hline No; & & $8 ;$ & $81 ;$ & & \\
\hline Yes & & 2 & 3 & & \\
\hline Time from symptom onset to presentation: & 94 & & & 0.193 & $2.567(0.621,10.604)$ \\
\hline$<15$ days; & & 3; & 44; & & \\
\hline$\geq 15$ days & & 7 & 40 & & \\
\hline ART initiation: & 94 & & & 0.421 & $1.731(0.455,6.582)$ \\
\hline No; & & $4 ;$ & 45; & & \\
\hline Yes & & 6 & 39 & & \\
\hline CD4+ T-cell counts: & 94 & & & 0.005 & $7.982(1.880,33.890)$ \\
\hline$\geq 25$ cells $/ \mu L_{;}$ & & $3 ;$ & $65 ;$ & & \\
\hline$<25$ cells $/ \mu \mathrm{L}$ & & 7 & 19 & & \\
\hline Patchy lesions on MRI: & 94 & & & 0.192 & $2.437(0.639,9.305)$ \\
\hline No; & & 4 & $52 ;$ & & \\
\hline Yes & & 6 & 32 & & \\
\hline Ring-enhancing lesions on MRI: & 94 & & & 0.790 & $0.833(0.218,3.188)$ \\
\hline No; & & $4 ;$ & 30 & & \\
\hline Yes & & 6 & 54 & & \\
\hline
\end{tabular}


Table 2 Multivariate logistic regression analysis and scoring variables

\begin{tabular}{llll}
\hline Variables & Coefficient & $\boldsymbol{p}$ & score \\
\hline Fever & 7.764 & 0.031 & 4 \\
Dizziness & 3.624 & 0.099 & 2 \\
Memory deficits & 3.066 & 0.07 & 2 \\
Disorders of consciousness & 5.065 & 0.033 \\
Time from symptom onset to presentation $\geq 15$ days & 3.34 & 0.048 \\
CD4+ T-cell counts $<25$ cells/ML & 5.431 & 0.011 & 2 \\
Lesions manifesting as patchy & 2.006 & 0.180 & 3 \\
\hline
\end{tabular}

\section{Multivariate logistic regression analysis and scoring of variables}

Ten variables had a $p$-value $<0.2$ from the results of univariate logistic regression analysis, and all of them were selected for multivariate logistic regression analysis. Finally, seven variables were retained in the optimal regression equation with $p<0.05$ through forward logistic regression stepwise regression analysis, and further incorporated for score assignment. Patchy brain lesions present on CT or MRI was the variable with the lowest regression coefficient (2.006), which means that "patchy brain lesions" as a risk factor, has the lowest influence on death, and was thus assigned the lowest arbitrary score of one. The remaining six variables were as follows: time from symptom onset to presentation, fever, dizziness, CD4+ T-cell counts, memory deficits and a

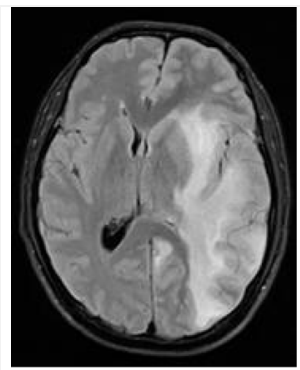

c

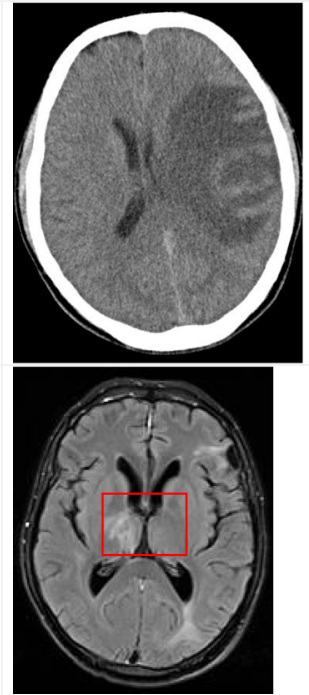

$\mathrm{b}$

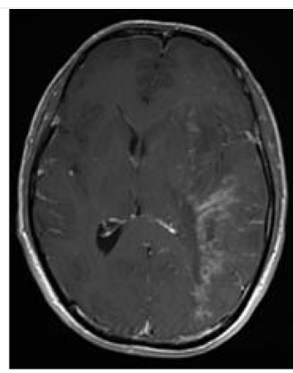

$\mathrm{d}$

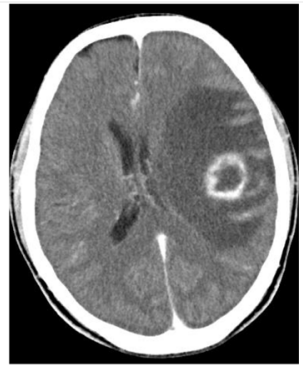

f

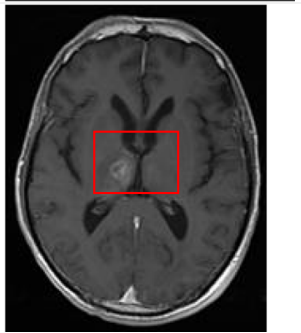

Fig. 1 Radiological images of three cases of non-survival. The T2 flair series showed large patchy abnormal signals on the left frontal lobe, temporal lobe, and occipital lobe (a). T1 gadolinium-enhanced scans showed multiple patchy enhancements in the left frontal lobe, temporal lobe, and occipital lobe (b). A CT scan of the head revealed a large patch of low-density shadow on the right temporal lobe (c), and an enhanced CT scan revealed a ring-shaped enhancement on the right temporal lobe (d). The T2 flair series showed slice-like and patch-like abnormal signals at the junction of the right thalamus, left frontal lobe, and left temporal and occipital lobes (e). A T1 gadolinium-enhanced scan showed a ring-shaped enhancement in the right thalamus (f). Note: Lesions on temporal lobe were reported to be related to memory deficits (ad); lesions on thalamus were reported to be related to disorders of consciousness (e-f). 
disorders of consciousness, and which were assigned a score in sequence, after dividing each regression coefficient of six different risk factors by 2.006 to determine the score (in units of 0.5), as shown in Table 2.

TE cerebral lesions on temporal lobe structures of non-survivor cases were assumed to be related to memory deficits [9], as shown in Fig. 1 a-d, and typical patchy lesions in the thalamus of one non-survivor, which was considered to be associated with disorders of consciousness [10], as shown in Fig. 1(e-f), respectively.

\section{Score distribution and ROC curve of the scoring model}

The score distribution of survivor and non-survivor cases of HIV-infected patients with TE is shown in Fig. 2a. In conjunction with our established model, as shown in Fig. $2 \mathrm{a}$, a criterion value of ' 9 ' was selected as the optimal cutoff value. The AUC of the ROC curve of this scoring model was $0.976(p<0.001)$, and the sensitivity and specificity of this scoring model at the cut-off value of "9" was $100.0 \%$ (95\%CI, 69.2-100.0\%) and 86.9\% (95\%CI, 77.8-93.3\%), respectively, as shown in Fig. 2b and c.

\section{Verification and accuracy of the scoring model}

Forty-five HIV-infected cases with TE were involved in the scoring model verification cohort, and the sensitivity and specificity of the model in this cohort was 81.8 and $94.1 \%$, respectively. The accuracy of the scoring system in the verification cohort was 91.1\% (41/45), as shown in Fig. 3.

\section{Discussion}

The prevalence of co-infection with Toxoplasma gondii and HIV ranges from 25.1 to $60.7 \%$ in different countries [11]. The mortality of HIV-associated TE during hospitalization, with appropriate anti-toxoplasma treatment, may be up to $30 \%$ [4]. The 6-week mortality in the present study was $11.7 \%$. In order to effectively reduce mortality in patients with HIV-associated TE, timely targeted intervention based on specific prognostic factors is a sensible undertaking. Thus, we conducted this study, and aimed to discover specific risk factors of 6-week mortality, and to establish a scoring system model to credibly predict poor outcomes in patients with HIV-associated TE. a

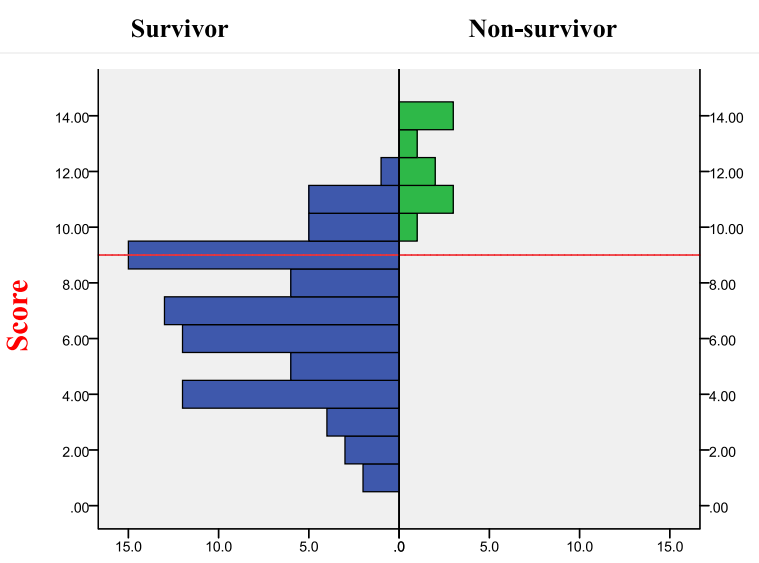

Frequency

Frequency $\mathrm{b}$

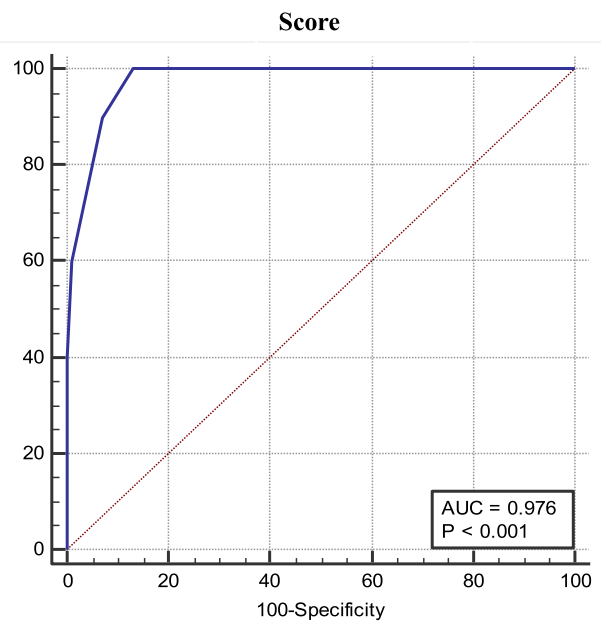

$\mathrm{c}$

\begin{tabular}{cccccccc}
\hline Criterion & Sensitivity & $\mathbf{9 5 \%}$ CI & Specificity & $\mathbf{9 5 \%}$ CI & \multicolumn{2}{c}{+ LR } & \multicolumn{1}{c}{-LR } \\
\hline$\geq 1$ & 100.00 & $69.2-100.0$ & 0.00 & $0.0-4.3$ & 1.00 & \\
$>9$ & 100.00 & $69.2-100.0$ & 86.90 & $77.8-93.3$ & 7.64 & 0.00 \\
$>10$ & 90.00 & $55.5-99.7$ & 92.86 & $85.1-97.3$ & 12.60 & 0.11 \\
$>11$ & 60.00 & $26.2-87.8$ & 98.81 & $93.5-100.0$ & 50.40 & 0.40 \\
$>12$ & 40.00 & $12.2-73.8$ & 100.00 & $95.7-100.0$ & & 0.60 \\
$>14$ & 0.00 & $0.0-30.8$ & 100.00 & $95.7-100.0$ & & 1.00 \\
\hline
\end{tabular}

Fig. 2 The development of the scoring model. (a) distribution of cases of survival and cases of non-survival in HIV-infected patients with TE; (b) ROC curve of the scoring model; (c) criterion values and coordinates of the ROC curve 


\section{Score}

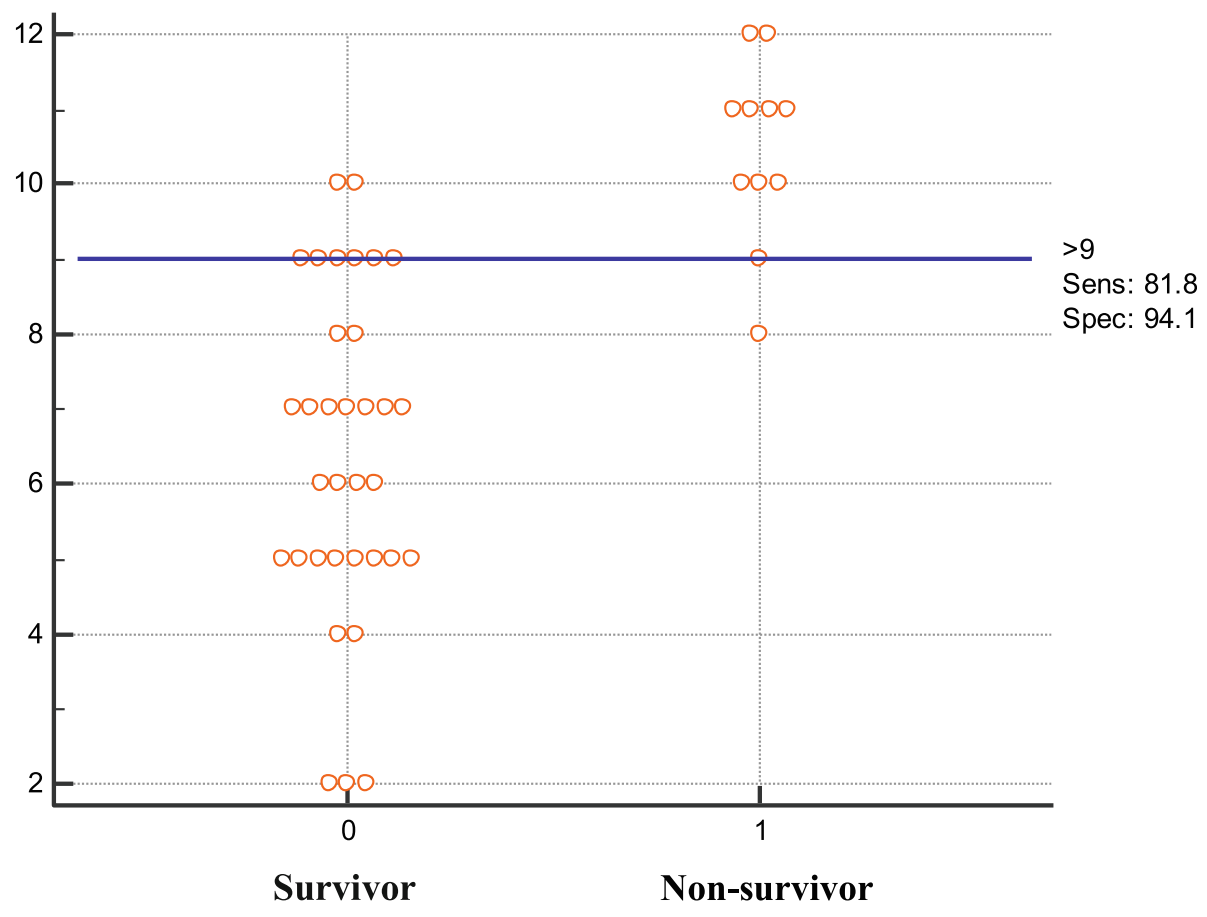

Fig. 3 Integral distribution of HIV-infected TE patients in the validation cohort

Thus far, few studies have described the relationship between proposed risk factors and poor outcomes among HIV-infected patients with TE. Libório et al. conducted a retrospective cohort study and found that AKI $(\mathrm{OR}=8.3)$ and hyponatremia $(\mathrm{OR}=9.9)$ were independent risk factors for death in HIV-infected patients with TE [5]. Another retrospective cohort study regarding patients with severe cerebral toxoplasmosis conducted by Sonneville et al., showed that a CD4+ T-cell counts $<25$ cells $/ \mu \mathrm{L}(\mathrm{OR}=2.7)$, and a GCS score $\leq 8(\mathrm{OR}=3.1)$ were independently associated with poor outcomes (modified Rankin Scale score $\geq 2$ ) at 3 months [6]. Conversely, CD8+ T-cell counts were reported to play a dominant role as a protective factor in AIDS patients with chronic toxoplasmosis [12]. Hoffmann et al. found that overall survival of AIDS patients with toxoplasmosis was significantly improved in those patients diagnosed after 1996 (the highly-active antiretroviral therapy era), those without a previous AIDS-defining illness, those aged $<45$ years, and those with a LDH level $<300$ $\mathrm{U} / \mathrm{L}[13]$.

Based on the results of these preceding studies and the integrity of the data in the present study, we selected 16 different potential risk factors as prognostic factors for screening and scoring model development. The results of univariate and multivariate logistic regression analysis revealed that fever and dizziness were protective factors in survivors of HIV-associated TE, suggesting that patients with these two encephalopathy-related symptoms are more likely to seek medical treatment expeditiously, thereby avoiding treatment delay and reducing mortality. In addition, the significant statistical difference in overall survival between the time from symptom onset to presentation $\geq 15$ days and $<15$ days $(p=0.048)$ in survivors and non-survivors, also reinforces the benefits of timeous treatment. We observed that CD4+ T-cell counts < 25 cells/ $\mu \mathrm{L}$ was associated with non-survival at 6 -weeks after diagnosis $(\mathrm{OR}=7.982)$ in HIV-infected patients with TE. Memory deficits usually manifested as episodic and semantic learning disability, and was reported to be related to damage of medial temporal lobe structures, and the hippocampus [8]. Previous studies have confirmed that at a neuroanatomic level, activation of the cerebral cortex occurs with passage of sensory data from the upper brainstem via the reticulo-thalamo-cortical and extrathalamic pathways [10]. Thus, toxoplasmic disorders of consciousness may be related to lesions of the thalamus and its surrounding tissues [10]. Patchy cortical lesions are usually scattered, usually involving multiple areas of the brain, and are considered to be associated with non-survival in this study. The present developed scoring model is based on data extracted from 94 retrospective case files, and is correlated with high sensitivity and specificity. The scoring system has also performed well when tested in the validation cohort. Also, in this newly-developed scoring system, simple risk factors that are readily available from patient clinical 
records allows for the seamless and expeditious implementation of the scoring system, allowing prognostication and consequent appropriate therapeutic interventions to be implemented in an expeditious manner.

There are a few limitations to the present study. Firstly, as a consequence of the widespread use of ART in China, only modest numbers of cases with complete data were involved in the scoring model development, and also in the model verification cohort. Secondly, due to the absence of adequate data for some risk factors, screening for risk factors has introduced an inherent degree of bias to the present study. Thirdly, this scoring model is based on Chinese HIV-infected patients with TE. Whether the scoring system is applicable to TE patients without HIV, or to other population groups requires further research. Lastly, long-term mortality and neuropsychological patient outcomes cannot be extrapolated from our findings.

\section{Conclusions}

The developed scoring system was established to evaluate specific risk factors influencing prognosis of HIV-infected patients with TE. The scoring system included seven risk factors, use of which allows expeditious application of accurate prognostication, and implementation of appropriate therapeutic interventions in HIV-infected patients with TE. However, whether the scoring system is applicable to TE patients without HIV, or to population groups other than Chinese, warrants further research.

\section{Supplementary Information}

The online version contains supplementary material available at https://doi. org/10.1186/s12879-020-05651-x.

Additional file 1: Supplementary Figure 1. Study Flow Diagram

\section{Abbreviations}

AIDS: Acquired immunodeficiency syndrome; TE: Toxoplasma encephalitis; HIV: Human immunodeficiency virus; AKI: Acute kidney injury; LDH: Lactate dehydrogenase; GCS: Glasgow coma scale; SPSS: Statistical Package for the Social Sciences; ROC: Receiver operating characteristic; AUC: Area under the curve; CT: Computed tomography; MRI: Magnetic resonance imaging; PCR: Polymerase chain reaction; ART: Antiretroviral therapy

\section{Acknowledgments}

The authors thank Hui Chen, PhD for statistical advice, and Vijay Harypursat, MD for language improvement and revision of the manuscript.

\section{Authors' contributions}

Study design: ML and YKC. Collection and interpretation of data: $Y L$ and YMZ, YLZ, ZSJ, TTY, YS and KL. Statistical analyses and drafting of manuscript: $Y L$ and YMZ. Radiology assessment: XYL. Review of manuscript for important intellectual content: YQL and YKC.

All authors have read and approved the manuscript.

\section{Funding}

This work was supported by the National Science and Technology Major Project of China of the 13th Five-Year Plan (2018ZX10302104). The funding body played no role in the design of the study, in the collection, analysis, and interpretation of data, and in the writing of the manuscript.

\section{Availability of data and materials}

The raw data may be requested from the first author and corresponding author, with administrative permissions of the review board of the Chongqing Public Health Medical Center, first author and corresponding author. All patient data has been de-identified.

Ethics approval and consent to participate

The present study was approved by the institutional review board of the Chongqing Public Health Medical Center (No. 2019-003-02-KY). The institutional review board waived the requirement for written informed consent, since the present study was retrospective, and all patient data were analyzed in anonymity.

Consent for publication

Not applicable.

\section{Competing interests}

All authors declare that the research was conducted in the absence of any commercial or financial relationships that could be construed as a potential conflict of interest.

\section{Author details}

${ }^{1}$ Division of Infectious Diseases, Chongqing Public Health Medical Center, 109 Baoyu Road, Shapingba, Chongqing, China. ${ }^{2}$ Department of medical imaging, Chongqing Public Health Medical Center, Chongqing, China. ${ }^{3}$ Division of Infectious Diseases, Beijing Youan Hospital, Capital Medical University, Beijing, China. ${ }^{4}$ Department of Infectious Diseases, Liuzhou People's Hospital, Liuzhou, Guangxi, China. ${ }^{5}$ Division of Infectious Disease, Chengdu Public Health Clinical Medical Center, Chengdu, Sichuan, China. ${ }^{6}$ Department of Infectious Diseases, The Sixth People's Hospital of Zhengzhou, Zhengzhou, Henan, China. 'Division of Infectious Disease, Longtan Hospital of Guangxi Zhuang Autonomous Region, Liuzhou, Guangxi, China.

Received: 29 August 2020 Accepted: 23 November 2020

Published online: 04 December 2020

\section{References}

1. Rouatbi M, Amairia S, Amdouni Y, Boussaadoun MA, Ayadi O, Al-Hosary AAT, et al. Toxoplasma gondii infection and toxoplasmosis in North Africa: a review. Parasite. 2019;26:6. https://doi.org/10.1051/parasite/2019006.

2. McFarland M, Bartlett M, Davis P. Toxoplasmic Encephalitis. Edition: 1, Chapter: 1, Publisher: Avid Science, Editors: Avid Science. Encephalitis; 2016. p. 2-52.

3. Panel on Opportunistic Infections in HIV-Infected Adults and Adolescents. Guidelines for the prevention and treatment of opportunistic infections in HIV-infected adults and adolescents: Recommendations from the Centers for Disease Control and Prevention, the National Institutes of Health, and the HIV Medicine Association of the Infectious Diseases Society of America. [EB/OL]. http://aidsinfo.nih.gov/contentfiles/Ivguidelines/adult_oi.pdf [accessed May 16, 2020].

4. Luma HN, Tchaleu BC, Mapoure YN, Temfack E, Doualla MS, Halle MP, et al. Toxoplasma encephalitis in HIV/AIDS patients admitted to the Douala general hospital between 2004 and 2009: a cross sectional study. BMC Res Notes. 2013;6:146. https://doi.org/10.1186/1756-0500-6-146.

5. Libório AB, Silva GB Jr, Silva CG, Filho FL, Neto AS, Okoba W, et al. Hyponatremia, acute kidney injury, and mortality in HIV-related toxoplasmic encephalitis. Braz J Infect Dis. 2012;16(6):558-63. https://doi.org/10.1016/j. bjid.2012.08.015

6. Sonneville R, Schmidt M, Messika J, Hssain AA, da Daniel S, Klein IF, et al. Neurologic outcomes and adjunctive steroids in HIV patients with severe cerebral toxoplasmosis. Neurology. 2012;79(17):1762-6. https://doi.org/10. 1212/WNL.0b013e3182704040.

7. The National Institutes of Health, and the HIV Medicine Association of the Infectious Diseases Society of America. Guidelines for the Prevention and Treatment of Opportunistic Infections in HIV-Infected Adults and Adolescents: Recommendations from the Centers for Disease Control and Prevention, [EB/OL].(2019/6/26)[2020/5/16] https://aidsinfo.nih.gov/ guidelines/html/4/adult-and-adolescent-opportunistic-infection/322/ toxoplasma-gondii-encephalitis. 
8. Vidal JE, Hernandez AV, de Oliveira AC, Dauar RF, Barbosa SP Jr, Focaccia R, et al. Cerebral toxoplasmosis in HIV-positive patients in Brazil: clinical features and predictors of treatment response in the HAART era. AIDS Patient Care STDs. 2005;19(10):626-34. https://doi.org/10.1089/apc.2005.19. 626.

9. Eapen BC, Georgekutty J, Subbarao B, Bavishi S, Cifu DX. Disorders of consciousness. Phys Med Rehabil Clin N Am. 2017;28(2):245-58. https://doi. org/10.1016/.jpmr.2016.12.003.

10. Wang ZD, Wang SC, Liu HH, Ma HY, Li ZY, Wei F, et al. Prevalence and burden of toxoplasma gondii infection in HIV-infected people: a systematic review and meta-analysis. Lancet HIV. 2017;4(4):e177-88. https://doi.org/10. 1016/S2352-3018(17)30005-X.

11. Landrith TA, Harris TH, Wilson EH. Characteristics and critical function of CD8+ T cells in the toxoplasma-infected brain. Semin Immunopathol. 2015; 37(3):261-70. https://doi.org/10.1007/s00281-015-0487-3.

12. Hoffmann C, Ernst M, Meyer P, Wolf E, Rosenkranz T, Plettenberg A, et al. Evolving characteristics of toxoplasmosis in patients infected with human immunodeficiency virus-1: clinical course and toxoplasma gondii-specific immune responses. Clin Microbiol Infect. 2007;13(5):510-5. https://doi.org/ 10.1111/j.1469-0691.2007.01683.x.

13. Augustinack JC, van der Kouwe AJ, Salat DH, Benner T, Stevens AA, Annese J, et al. H.M.'s contributions to neuroscience: a review and autopsy studies. Hippocampus. 2014;24(11):1267-86. https://doi.org/10.1002/hipo.22354.

\section{Publisher's Note}

Springer Nature remains neutral with regard to jurisdictional claims in published maps and institutional affiliations.

Ready to submit your research? Choose BMC and benefit from:

- fast, convenient online submission

- thorough peer review by experienced researchers in your field

- rapid publication on acceptance

- support for research data, including large and complex data types

- gold Open Access which fosters wider collaboration and increased citations

- maximum visibility for your research: over $100 \mathrm{M}$ website views per year

At $\mathrm{BMC}$, research is always in progress.

Learn more biomedcentral.com/submissions 